University of Nebraska - Lincoln

DigitalCommons@University of Nebraska - Lincoln

Faculty Publications - Textiles, Merchandising and Fashion Design

Textiles, Merchandising and Fashion Design,

Department of

2022

Pilot-scale spinning and sucrose-tetra-aldehydes-crosslinking of feather-derived protein fibers with improved mechanical properties and water resistance

Bingnan $\mathrm{Mu}$

Faqrul Hassan

Qianmei Wu

Yiqi Yang

Follow this and additional works at: https://digitalcommons.unl.edu/textiles_facpub

Part of the Bioresource and Agricultural Engineering Commons, and the Polymer and Organic Materials Commons

This Article is brought to you for free and open access by the Textiles, Merchandising and Fashion Design, Department of at DigitalCommons@University of Nebraska - Lincoln. It has been accepted for inclusion in Faculty Publications - Textiles, Merchandising and Fashion Design by an authorized administrator of DigitalCommons@University of Nebraska - Lincoln. 


\title{
Pilot-scale spinning and sucrose-tetra-aldehydes-crosslinking of feather-derived protein fibers with improved mechanical properties and water resistance
}

\author{
Bingnan $\mathrm{Mu},{ }^{1}$ Faqrul Hassan, ${ }^{1}$ \\ Qianmei $\mathrm{Wu}^{2}{ }^{2}$ and Yiqi Yang 1,3,4
}

1 Department of Textiles, Merchandising and Fashion Design, 234, HECO

Building, University of Nebraska-Lincoln, Lincoln, NE 68583-0802

2 Department of Statistics, 340, Hardin Hall North Wing, University of Nebraska-Lincoln, Lincoln, NE 68583-0963

3 Department of Biological Systems Engineering, 234, HECO Building, University of Nebraska-Lincoln, Lincoln, NE 68583-0802

4 Nebraska Center for Materials and Nanoscience, 234, HECO Building,

University of Nebraska-Lincoln, Lincoln, NE 68583-0802

Corresponding author - Y. Yang, email yyang2@unl.edu

\begin{abstract}
Pliable and water-resistant protein fibers from feathers were developed via continuous one-step spinning and crosslinking using sucrose-tetra-aldehydes. Though flexible protein fibers from feathers were developed, poor wet stability and low tenacity still limit the large and high-end applications of feather-derived fibers. Despite that saccharide aldehydes are considered as an efficient and sustainable crosslinker, substantial concerns such as generation of formaldehyde, high consumption
\end{abstract}

Published in Sustainable Materials and Technologies 31 (2022) e00367

doi:10.1016/j.susmat.2021.e00367

Copyright $\odot 2021$ Elsevier B.V. Used by permission.

Submitted 29 December 2020; revised 10 July 2021; accepted 20 November 2021;

published 24 November 2021. 
of modifiers, loss of mechanical properties, yellowing, and decline in dyeability of fibers, exist after crosslinking. In this work, we controlled structures of crosslinkers and incorporated disaccharide-tetra-aldehydes into a continuous wet-spinning line. No formaldehyde was generated in the whole process. Protein fibers with controlled aldimine crosslinkages had not only a $92 \%$ tenacity retention after immersed in water for 1 week but also a substantial increase in mechanical properties. The dry and wet tenacity of feather-derived fibers was $120 \%$ and $90 \%$ of wool, respectively. Moreover, crosslinking from disaccharide-tetra-aldehydes retained the color of fibers and minimized the consumption of amine groups, the dyeing sites, on keratin. No toxicity was introduced by crosslinking. This work promotes resource recovery from poultry wastes, decreases the reliance of fiber industry on petroleum-based products, and diminishes environmental impact of fiber industries.

Keywords: feather wastes, fiber production, sucrose-tetra-aldehydes crosslinking, wet-stability, formaldehyde

\section{Introduction}

Regeneration of protein fibers from poultry wastes is very promising because of the environmental $\&$ health threat of feathers, huge global demand for natural fibers, and unique properties of feathers. Raw poultry feathers contaminated with blood, feces and bacterial are considered hazardous wastes [1,2]. For example, when contaminated with bacteria, feathers were odoriferous and unfit for further valorization [3]. Moreover, the US Environmental Protection Agency found out that poultry wastes, including feathers are one of the highest contributors of nitrogen [4]. Also, inhalable feather dust contains several allergenic components [5]. For instance, chicken serum albumin (alphalivetin) has been implicated as the causative allergen of Bird-Egg Syndrome. Therefore, in the UK, poultry dust including feathers is treated as a substance hazardous to health and under the Control of Substances Hazardous to Health Regulations (COSHH) [6]. Considering the health and environmental concerns, feather-containing wastes from the poultry industry have to be properly managed and prevent the contamination of bacteria, yeast, fungi, and virus and emission of odors $[7,8]$. Though various technologies have been developed to tackle feather pollution [9], feather processing is still a problem because of the low profit margin. Because of unique properties [10-14], utilization of feathers has huge value-additions $[15,16]$ and promotes sustainable decontamination of poultry wastes. 
Though our group has recently continuously spun flexible keratin fibers from feathers [17], poor wet properties and low property retention after wet treatment still limit large-scale applications of feather-derived fibers [18]. Establishment of additional crosslinkages in regenerated fibers would potentially improve wet properties of fibers. Considering that the proportion of amino acids containing amines as the side group in the keratin is as high as $8 \%$ [19], it is feasible to crosslink keratin fibers with aldehyde-based crosslinkers since aldehyde crosslinking requires low crosslinking temperatures and cause fewer damages to proteins. Though saccharide aldehydes were proven to be an efficient biobased crosslinker for various protein materials, there are still concerns with this crosslinker developed by previous methods [20]. We found the formation of formaldehyde during the oxidation of saccharides. Also, sucrose-tetra-aldehydes, the desirable crosslinker, were not the dominant products in saccharide aldehydes using the previous recipe [21]. As a result, an excessive amount of saccharide aldehydes, e.g. $6 \%-10 \%$ were added into protein matrix. $[22,23]$ and consumed a large number of amine groups on keratin. Consequently, the treated fibers might be undyeable, not to mention the dyeing dark shades. Besides, to achieve desirable crosslinking performances, high temperature might be to be used using saccharide aldehydes. As a result, final products had a substantial decrease in breaking elongation and compressibility [23]. Furthermore, uncontrolled crosslinking using saccharide aldehydes led to substantial color change and yellowing [22]. For fibers obtained through a high degree of drawings, the molecular arrangement is relatively regular, and the length between the backbone molecules is relatively uniform. Therefore, the subtle change in the structure of the crosslinking agent would remarkably affect properties of fibers. Plus, ensuring the fast and full crosslinking on a continuous line is a problem [24].

In this paper, formaldehyde-free crosslinkers with control of structures of saccharide aldehydes and crosslinking process using such crosslinkers were developed. Feather-derived fibers were continuously spun with the incorporation of formaldehyde-free sugar-based aldimine crosslinkages. Keratin fibers after crosslinking had good property retention after aqueous treatment, improved mechanical properties, and unchanged color and cytocompatibility. Also, considerable 
dyeing sites on keratin fibers were retained after crosslinking. Substantial value additions were demonstrated to fiber regeneration, which has a positive environmental impact.

\section{Experimental}

\subsection{Materials}

Sucrose (99.9\%), raffinose (99.9\%), were purchased from VWR International (Radnor, PA, US). Sodium periodate (98\%) was purchased from Alfa Aesar (Haverhill, MA, US), 2,4- dinitrophenylhydrazine (97\%) was purchased from Aldrich (St. Louis, MO, US). Feathers were provided by Feather fiber corporation, Nixa, CO, with keratin content approximately $92 \%$. Chemical reagents used in SDS-PAGE analysis, including LDS sample buffer $(4 \times)$, Nupage $20 \times$ MES running buffer and NuPAGE 4-12\% Bis-Tris gel, were purchased from Invitrogen, Inc. (Carlsbad, CA, US).

\subsection{Preparation of crosslinkers}

Sucrose and raffinose were oxidized by sodium periodate at room temperature for $5 \mathrm{~h}$ to obtain disaccharide-tetra-aldehyde and trisaccharide-tetra-aldehydes, respectively. The $\mathrm{pH}$ of reaction solution was kept at $6.0 \pm 0.1$ using $1 \mathrm{M} \mathrm{NaOH}$ solution. Several droplets of $\mathrm{NaOH}$ solution were added to the reaction medium to keep $\mathrm{pH}$ unchanged. To obtain the highest portion of disaccharide-tetra-aldehyde and trisaccharide-tetra-aldehydes from oxidation products, the molar ratios of sucrose and periodate, and raffinose and periodate were 1:3 and $1: 4$, respectively. After the reaction, slightly excessive barium dichloride was added to completely precipitate the periodates. The mixture was filtrated to obtain the saccharides derivatives. For validation of the full removal of oxidants, a potassium iodide-starch solution was used. Sodium sulfite was to further remove the residual oxidants.

\subsection{Determination of structures of saccharide derivatives}

Oxidized saccharides solutions after complete removal of oxidation agents were divided into two parts, one for determination of ratios 
of saccharide derivatives with different sugar rings oxidized and the other part for determining molecular weights of each structure. The first part was further hydrolyzed overnight under $60^{\circ} \mathrm{C}$ after the addition of $3 \mathrm{M} \mathrm{HCl}$ to cleave all glycosidic bonds. The obtained solution was derivatized with 2,4- dinitrophenylhydrazine for better detection in HPLC. The second part was directly derivatized with 2,4- dinitrophenylhydrazine to convert all aldehyde groups to the Schiff base. For the HPLC-MS analysis, a C-18 reversed-phase column (Acclaim 120, $120 \AA, 4.6 \times 250 \mathrm{~mm}, 5 \mu \mathrm{m})$ and a UV-Vis detector were equipped in the HPLC system. The samples $(25 \mu \mathrm{L})$ were injected and eluted at a flow rate of $1 \mathrm{~mL} / \mathrm{min}$ at $30^{\circ} \mathrm{C}$. A mixture of solvents water-acetonitrile $(55: 45 \mathrm{~V} / \mathrm{V})$ was used as the mobile phase. The UV-Vis detector was set at $360 \mathrm{~nm}$. The MSQ Plus MS was controlled by Xcalibur software using the ESI mode with positive polarity at a probe temperature of $350{ }^{\circ} \mathrm{C}$ and a cone voltage of $60 \mathrm{~V}$. The detector was set to scan the range of $200-2000 \mathrm{~m} / \mathrm{z}$.

\subsection{Determination of length distribution between two aldehyde groups on saccharide derivatives}

The structures of saccharides analyzed by HPLC-MS were optimized using Gaussian 09 package. Specifically, DFT calculations were performed by using the M06-2× density functional and the 6-31+G* basis set. Unless stated otherwise, solvent effects were included in all reported energies and treated by conductor-like polarizable continuum model (CPCM). After the optimization, lengths between aldehydes were obtained. The distribution of length between aldehyde from each sugar oxidation condition were obtained via $\mathrm{R}$ Studio version 1.4 (Boston, MA). Length distributions between aldehyde from each structure of saccharide aldehydes were obtained using Prop function. The combined distribution between aldehydes from multiple structures developed by each sugar oxidation conditions was obtained using Runif and Ggplot function.

\subsection{Preparation of spinning dope}

Detailed keratin extraction and spinning were shown in our previous work [17]. Briefly, $2 \mathrm{M}$ urea along with 10\% SDS and 10\% based on the weight of feathers was used for the keratin extraction, which was 
under $70{ }^{\circ} \mathrm{C}$ for $12 \mathrm{~h}$ with $\mathrm{pH}$ adjusted to 10.5 . For the preparation of spinning dope, $27 \%$ keratin extracts and $2 \%$ mercaptoethanol, as well as $10 \%$ SDS based on the keratin weight, were added into carbonate buffer with $\mathrm{pH}$ 8. The fiber was spun via stepwise drawing and oxidation, as shown in our previous work.

\subsection{Continuous spinning with crosslinking}

A continuous wet spinning line (ALEX JAMES AND ASSOC, Greenville, SC, US) was used for spin and crosslink keratin fibers, as shown in Fig. 1. The keratin spinning dope was centrifuged before being installed onto the spinning line. Keratin solution was extruded via a spinneret to the coagulation bath containing $15 \mathrm{wt} \% \mathrm{Na}_{2} \mathrm{SO}_{4}$ and 5 $\mathrm{wt} \% \mathrm{ZnSO}_{4}$. The $\mathrm{pH}$ of the coagulation bath was adjusted to 2 using an acetate buffer. Then filaments went through multiple drawing rollers and oxidation baths containing $4 \mathrm{~g} \mathrm{~L}^{-1}$ sodium periodate to become finer with high recovery of disulfide crosslinkages. The $\mathrm{pH}$ of oxidation baths was adjusted to 2 using an acetate buffer. After the step of multiple drawings and oxidation for formation of disulfide crosslinkages, as shown in Fig. 1, filaments underwent multiple dippings and squeezings for the establishment of crosslinkages brought by saccharide aldehydes. The $\mathrm{pH}$ of the dipping solution, containing saccharide aldehydes, was adjust to 5 with acetic buffer. Concentrations of saccharide aldehydes in fibers were controlled by squeezings. Filaments with desirable amount saccharide aldehydes reached the heating roller to form aldimine crosslinkages in fibers. The temperature of the heating roller was controllable for optimized crosslinking.

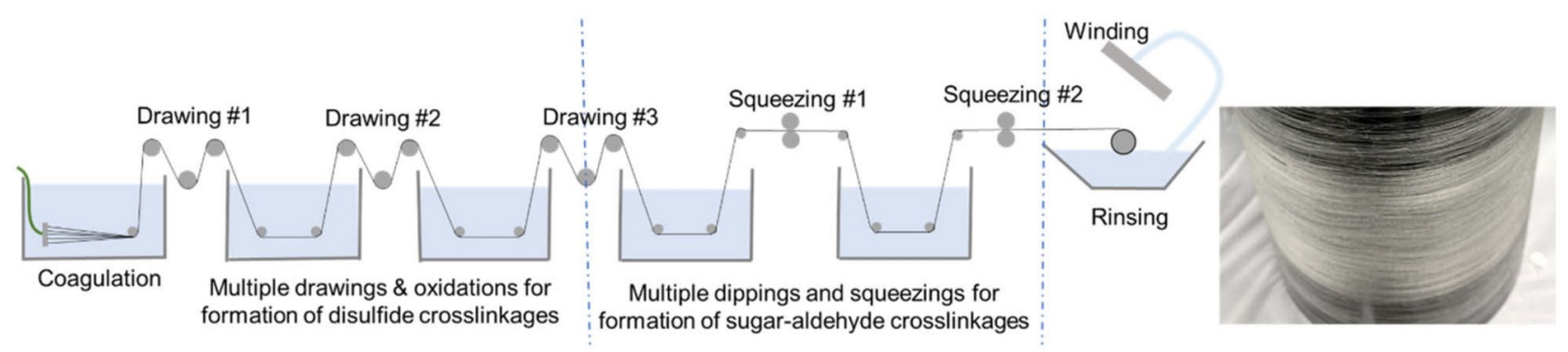

Fig. 1. Diagram of continuous spinning and formyl-saccharide-crosslinking of keratin fibers. Continuously spun and crosslinked keratin filaments are shown on the right side of the fig. 
Finally, filaments were washed in a bath with $\mathrm{pH}$ of 4 adjusted by acetic acid. For a comparison, citric acid as a control crosslinker was used to crosslink spun keratin fibers using the method from our previous work [25]. Briefly, the crosslinking bath contained $22 \%$ citric acid with $\mathrm{pH} 8$ adjusted by $\mathrm{NaOH}$. The crosslinking was conducted at $50{ }^{\circ} \mathrm{C}$ for $2.5 \mathrm{~h}$. The dry fibers obtained were dried at $85^{\circ} \mathrm{C}$ for $1 \mathrm{~h}$ and later $125^{\circ} \mathrm{C}$ for another $1 \mathrm{~h}$. Supplementary video shows actual spinning and crosslinking of keratin filaments.

\subsection{Molecular weight of crosslinked keratin by saccharide aldehydes}

About $1 \mathrm{mg}$ of feather and keratin fiber was dissolved in $100 \mu \mathrm{L}$ of NuPAGE LDS sample buffer $(1 \times)$ with mercaptoethanol. The addition of reducing agents was to exclude the interference of disulfide crosslinkages. The solution was centrifuged before loading. The molecular weights of the protein standard mixture ranged from 3 to $188 \mathrm{kDa}$.

\subsection{Mechanical properties}

For mechanical properties in dry state, fibers were conditioned at 21 ${ }^{\circ} \mathrm{C}$ and $65 \% \mathrm{RH}$ for $24 \mathrm{~h}$ before tests. For mechanical properties in wet state, fibers were immersed in water for 30 min before the test. For wet stability tests, fibers were immersed in water with $\mathrm{pH} 5$ at $35^{\circ} \mathrm{C}$ for 1 week. After that, the fibers were washed and annealed at $125^{\circ} \mathrm{C}$ for $1 \mathrm{~h}$. ASTM standard D-3822 was used to measure tensile properties of keratin fibers on an Instron tensile testing machine (Norwood, MA). Gauge length set for testing was $1 \mathrm{in}$. with speed of $18 \mathrm{~mm} / \mathrm{min}$.

\subsection{Cytocompatibility tests}

The growth state of NIH3T3 cell assessed the cytocompatibility of keratin with and without saccharide-aldehyde crosslinking. Samples were sterilized at $120^{\circ} \mathrm{C}$ for $60 \mathrm{~min}$ before cell culture. The cells were incubated onto the samples and cultured at $37^{\circ} \mathrm{C}$ for 3 days. Then, the samples were washed with PBS, and placed in a 24-well plate. The dyeing buffer contained $10 \mu \mathrm{L}$ Calcein-AM and $10 \mu \mathrm{L}$ PI-DNA in $10 \mathrm{~mL}$. Subsequently, the NIH3T3 cells cultured on samples were observed under a fluorescent microscope (Leica DMI8, Buffalo Grove, IL). The 
optical density of the liquid was determined at $490 \mathrm{~nm}$ using a fluorescent microscope.

\subsection{Statistical analysis}

One-way analysis of variance using the Scheffe test with a confidence interval of $95 \%$ was used for all obtained data. The statistical analysis was conducted on SAS 9.4 software (Cary, North Carolina) and survey procedures of PROC GLIMMIX.

\section{Results and discussion}

\subsection{High yield of formaldehyde-free sucrose-tetra-aldehydes}

Fig. 2 shows the pH effect on the formation of sucrose-tetra-aldehydes and formaldehyde during the sugar oxidation process. Results show that keeping $\mathrm{pH}$ at 6 provided the highest oxidation degree (total aldehydes) and amount of sucrose-tetra-aldehydes without the

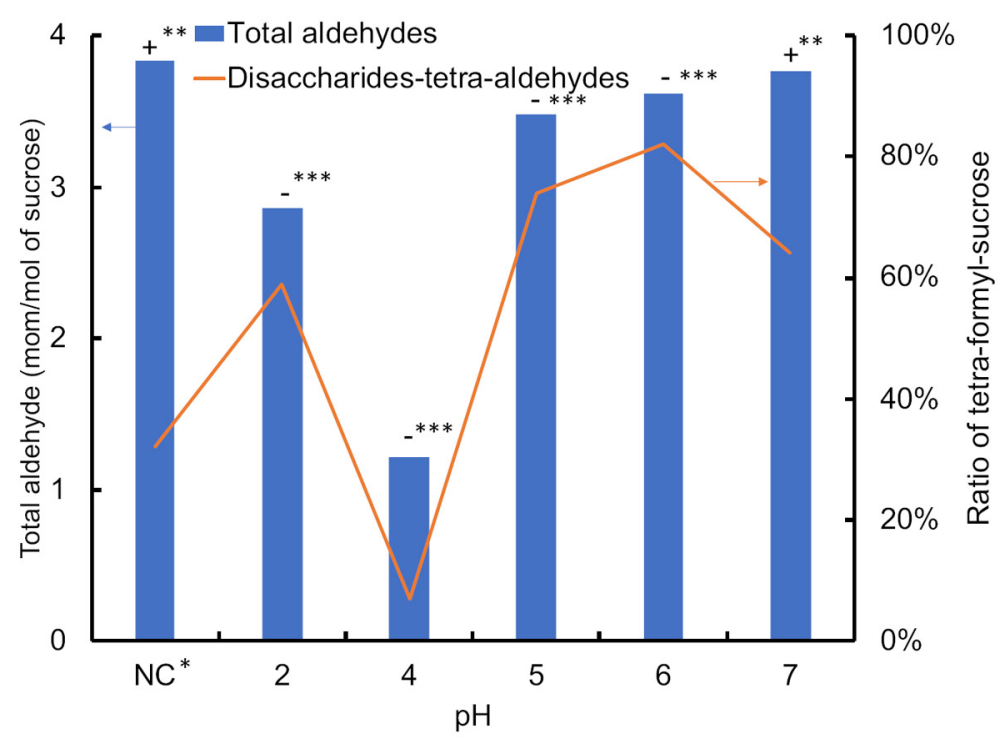

Fig. 2. The pH effect on total aldehydes, ratio of sucrose-tetra-aldehydes, and formation of formaldehyde. (Molar ratio sucrose and periodate was 1:3. Reaction time was $5 \mathrm{~h}$. The detection limit for formaldehyde was $0.1 \mathrm{ppm}$ ). Footnote: ${ }^{*} \mathrm{NC}=$ uncontrolled $\mathrm{pH}$, the final $\mathrm{pH}$ was $0.87 ;^{* *}+$ : detection of formaldehyde; ${ }^{* *}-$ : no detection of formaldehyde. 
formation of formaldehyde. It was found that periodate at $\mathrm{pH} 4$ had the lowest oxidation performance, resulting in the lowest total aldehyde amount and ratio of sucrose-tetra-aldehyde. An increase in $\mathrm{pH}$ would lift the oxidation power of periodate, which not only improves the total aldehyde amount but also synchronously oxidized both sugar rings. However, the oxidation power was needed to be contained, or formation of formaldehyde and a decrease in the portion of sucrosetetra-aldehydes occurred due to the overoxidation. The results also agree with the previous results that too much oxidation power of periodate contributed to a high degree of overoxidation [26]. Finally, a $\mathrm{pH}$ of 6 was selected to ensure the formation of formaldehyde-free sucrose-tetra-aldehydes.

\subsection{Control of length of crosslinkages}

Fig. 3 shows the difference in length of crosslinkages brought by two sets of saccharide-aldehydes. Previous studies demonstrated that rigid and flexible crosslinkers contributed to improving the breaking strength and strain of cellulosic products, respectively [27]. However, the length of crosslinkages brought by flexible crosslinkers should also affect the mechanical properties of protein fibers. Here, two kinds of flexible saccharides, sucrose and raffinose were selected as disaccharide and trisaccharide. Major structures of saccharide aldehydes were determined by HPLC-MS. Molecular weights of major structures of saccharide aldehydes derivatized by 2,4- dinitrophenylhydrazine were shown in Fig. 3 b-d. For disaccharide-tetra-aldehyde, the length of crosslinkages, shown as the length between aldehydes can be extended to 8A. To further increase the length between aldehydes, saccharides with more sugar rings were needed. Raffinose, containing three sugar rings, was selected. After oxidation, the length between aldehydes can reach $14 \mathrm{~A}$ in the products.

\subsection{Substantial improvement in wet $\&$ dry properties}

Fig. 4 shows low temperature and a low amount of crosslinkers brought substantial improvement for mechanical properties of continuously spun keratin fibers within a short period. The results in Fig. 4a show that a moderate increase in crosslinking temperature can 

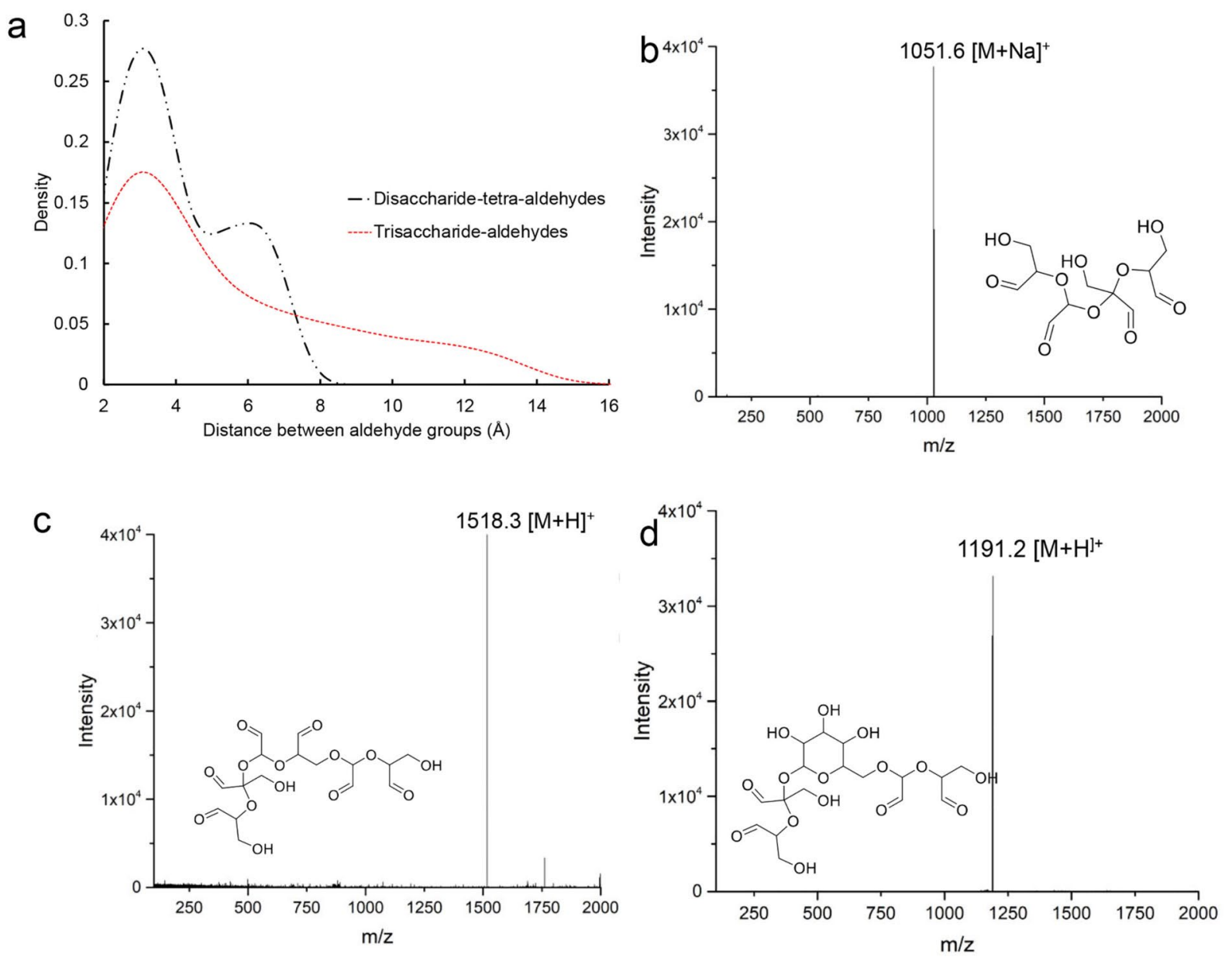

Fig. 3. a) Length distributions between aldehydes from different oxidation conditions. (Density obtained via Probability Density Function) b,c,d) Mass spectra of sucrose-tetra-aldehydes and raffinose-aldehydes ((Electrospray ionization mode with positive polarity at a probe temperature of $350^{\circ} \mathrm{C}$ and a cone voltage of $60 \mathrm{~V}$ using MSQ Plus MS). The aldehyde groups on saccharides were derivatized with 2,4-dinitrophenylhydrazine. The mass value was the saccharides derivatized with 2,4-dinitrophenylhydrazine. For sucrose-aldehydes, the ratio of sucrose-tetra-aldehyde was $81 \%$, for raffinose-aldehyde, the ratios of raffinose-tetra-aldehyde and raffinose-hexa-aldehydes were $40 \%$ and $25 \%$, respectively.

help improve the mechanical properties of fibers. For crosslinking under room temperature, the strength of keratin fiber increased slightly with the increase in crosslinking time, from $142 \mathrm{MPa}$ to $150 \mathrm{MPa}$. However, there was no significant difference. Meanwhile, at $50{ }^{\circ} \mathrm{C}$ after 1 $\mathrm{h}$ of crosslinking, the breaking stress of fibers can reach more than $200 \mathrm{MPa}$. As a further increase in crosslinking time, the mechanical 


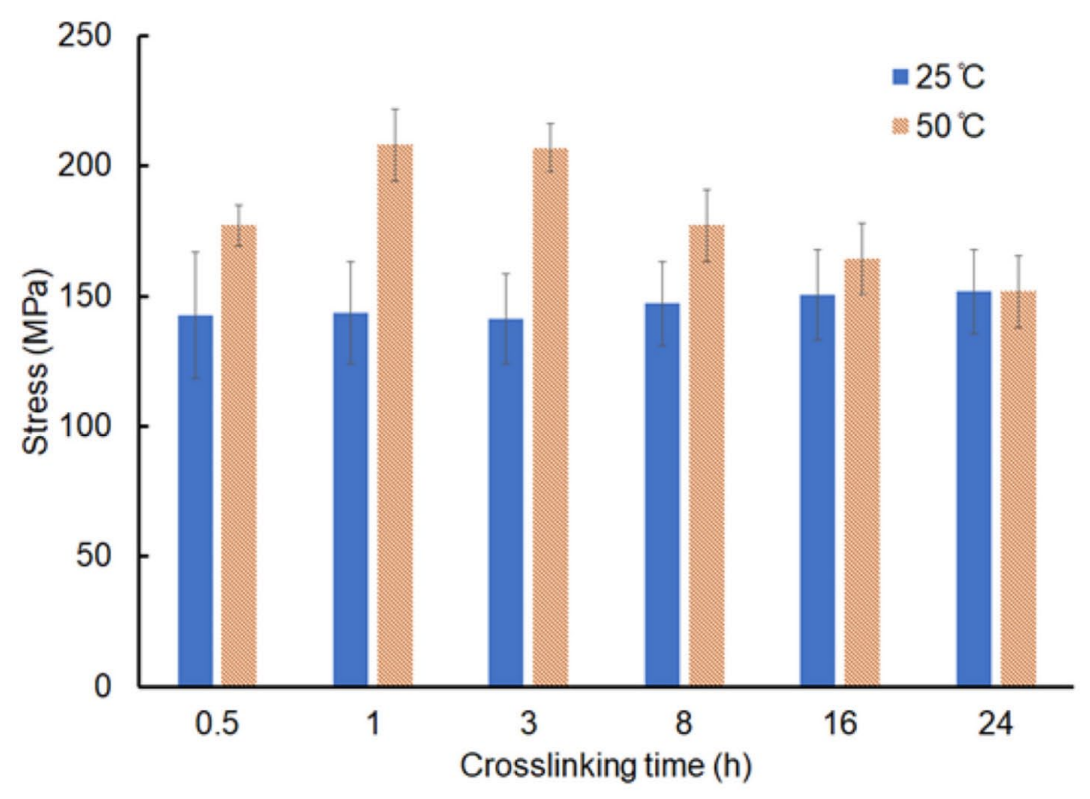

a

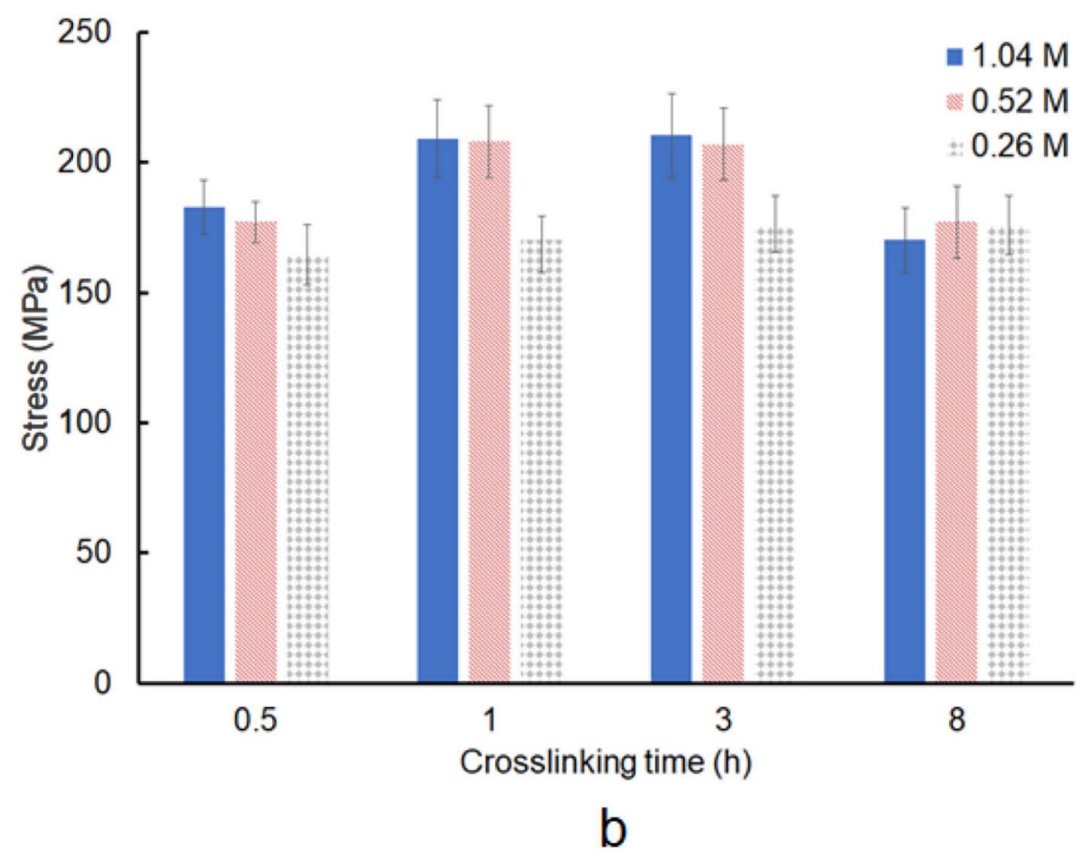

Fig. 4. a) Effect of temperatures and b) concentrations of saccharide derivatives (aldehyde concentration in solution) on mechanical properties of keratin fibers. The saccharide derivative was SU3, obtained by oxidizing sucrose by the 3-fold molar amount of sodium periodate. For a), crosslinking bath contained $0.52 \mathrm{M}$ aldehyde; Wet pick-up on fibers was $50 \%$. 
properties of the fibers gradually decreased, which may be due to over crosslinking. Over crosslinking would limit the movement of the keratin molecular chain, create an imbalanced tension within molecular chains and generate many weak points in fibers. As a result, fibers easily break when exerted external force. Fig. $4 \mathrm{~b}$ investigates the effect of concentrations of aldehydes in saccharide derivatives on the crosslinking properties in the oxidation bath. The results show that when the aldehyde group concentration was higher than $0.52 \mathrm{M}$ (around $2 \mathrm{wt} \%$ of crosslinker on keratin), desirable properties can be obtained after fiber crosslinking. For crosslinking time less than $3 \mathrm{~h}$, keratin fibers had higher breaking stress as the aldehyde concentrations were 1.04 and $0.52 \mathrm{M}$, than that as aldehyde concentration was $0.26 \mathrm{M}$. Higher aldehyde concentration ensured the fast-crosslinking reactions within a short time. When the crosslinking time was $8 \mathrm{~h}$, there was no significant difference in breaking stress of keratin fibers among three aldehyde concentrations. Possible reasons excessive long crosslinking time led to the over crosslinking of keratin molecules. Finally, $0.52 \mathrm{M}$ of aldehyde concentration was used for the future engineered crosslinking. Saccharide aldehydes were better than other biobased crosslinkers like polycarboxylic acid because saccharide aldehydes need a lower temperature to crosslink protein materials. Moreover, a weak alkali condition was required for polycarboxylic acid to crosslink protein. However, protein materials were unstable under alkalinity [28].

Fig. 5 shows sucrose-tetra-aldehydes had a better performance for improving wet and dry strength of keratin fibers. Fig. 5a shows the stress-strain curves of keratin fibers before and after engineered crosslinking using sucrose-tetra-aldehydes. There was a remarkable difference between curves with and without crosslinking using saccharide aldehydes after the yield point. After crosslinking using saccharide aldehydes, breaking strength and breaking strain of keratin fibers increased substantially. For keratin fibers without crosslinking using saccharide aldehydes, due to relatively low degree of disulfide crosslinkages (around 70\% recovery ratio of cystine [17]), interactions between protein molecules were low. As a result, tensile strength of fibers after the yield point decreased as molecules slippage to a high degree. With additional crosslinkages brought by saccharide aldehydes, backbones of protein were elongated and interactions between protein were strengthened. Elongated protein backbones promoted 


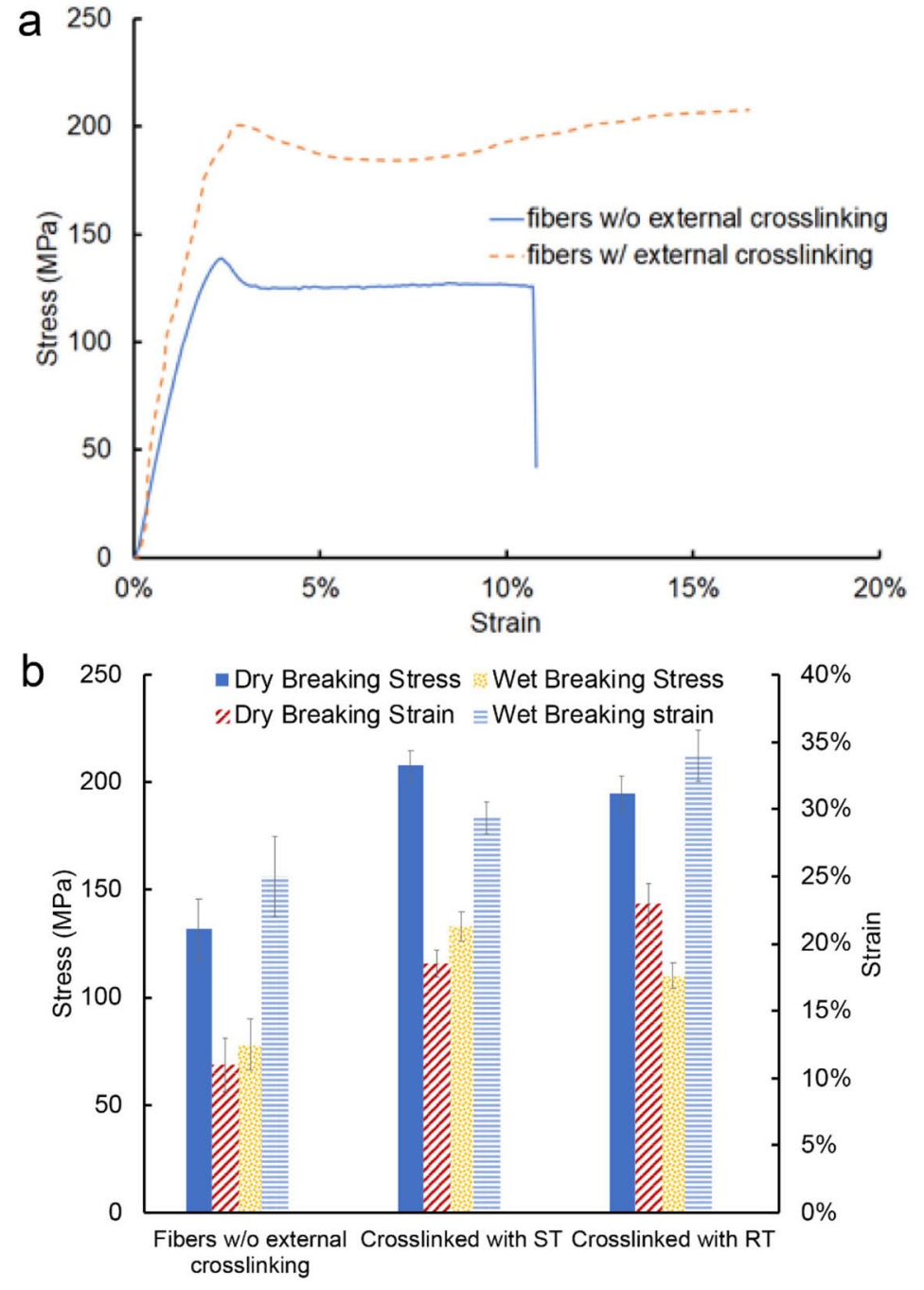

C
Fig. 5. a) Typical stress-strain curve for keratin fibers with and without crosslinking using saccharide aldehydes from continuous spinning line. Sucrose-tetra-aldehyde was used as the crosslinker. b) Dry and wet mechanical properties of keratin fibers before and after saccharide aldehydes crosslinking (The crosslinking was conducted under 50 ${ }^{\circ} \mathrm{C}$ for $1 \mathrm{~h}$ with aldehyde concentration of $0.52 \mathrm{M}, \mathrm{ST}=$ sucrose-tetra-aldehyde, RT = raffinose-tetra/hexa-aldehyde) c) Reducing SDS-PAGE of fibers with and without aldehyde crosslinking. Lanes $1-2$ : keratin crosslinked by sucrosealdehydes and raffinosealdehydes, and regenerated keratin without external crosslinking. The aldehyde concentrations for each crosslinking condition were $0.52 \mathrm{~mol} / \mathrm{L}$. The crosslinking conducted at $50^{\circ} \mathrm{C}$ for $1 \mathrm{~h}$.

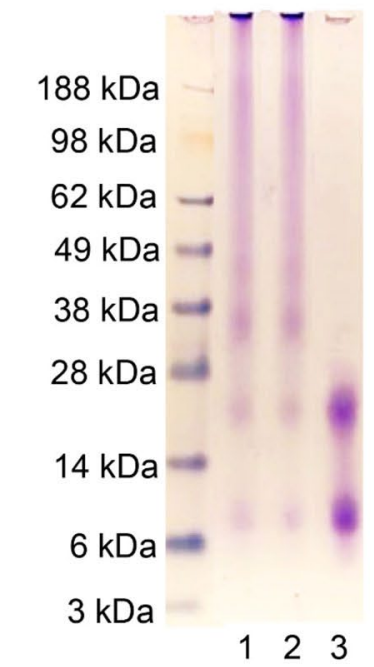


slippages between protein chains and strengthened interactions between protein chains increased the overall orientation of protein chains via stretching. Therefore, both breaking strength and strains of keratin fibers substantially increased with crosslinkages brought by saccharide aldehydes.

Fig. $5 b$ shows the effects of length of crosslinkages brought by saccharide aldehydes on mechanical properties of keratin fibers spun from a continuous line. Two kinds of crosslinkers, sucrose-tetra-aldehyde, and raffinose-aldehydes with remarkable differences in length of crosslinkages were used for keratin fibers. The results showed that after crosslinking, breaking stress and strain of keratin fibers under dry and wet states were substantially improved. With sucrose-tetraaldehyde crosslinking, breaking stress at dry and wet states increased by $58 \%$ and $71 \%$, respectively while breaking strains at dry and wet states increased by $72 \%$ and $16 \%$, respectively. With raffinose-aldehydes crosslinking, breaking stress at dry and wet states increased by $47 \%$ and $41 \%$, respectively while breaking strains at dry and wet states increased by $110 \%$ and $36 \%$, respectively. According to the results of Fig. 3a, compared to sucrose-aldehydes, length distributions between aldehydes of raffinose-aldehydes were large, i.e. higher than $8 \mathrm{~A}$. With raffinose-aldehyde crosslinking, the average length of crosslinkages would be longer than the crosslinkages formed by sucrosealdehyde. The long crosslinkages made slippage between protein molecular chains easier. As a result, the final breaking strain was high for raffinose-aldehyde crosslinking, but breaking stress was lower than that using sucrose-aldehyde. Compared to polycarboxylic acid, commonly used biobased crosslinker, saccharide-based aldehydes had better capability to improve mechanical properties of protein materials because saccharide aldehydes had high reactivity and required lower temperature. As a result, less damage would be brought to protein materials by saccharide aldehydes. For example, mechanical properties of silk crosslinked with citric acid decreased by $20 \%$ [29]. Some studies used low temperature for citric acid crosslinking. Finally, strength of protein materials at wet and dry states only improved by $5 \%$ and $16 \%$, respectively, substantially lower than improvement brought by saccharide-aldehydes [28].

Fig. $5 c$ shows similar crosslinking degrees between sucrose-aldehyde and raffinose-aldehyde crosslinking, further demonstrating that 
difference in mechanical properties of fibers resulted from the length of crosslinkages. The results show that the molecular weight of keratin significantly increased after crosslinking with saccharide aldehydes. After crosslinking with sucrose-aldehyde and raffinose-aldehyde, the original molecular bands of the keratin backbone were almost disappeared, indicating that most keratin backbones were crosslinked.

\subsection{Improved water resistance of crosslinked fibers}

Fig. 6 shows high retention of fiber strength after aqueous treatment after establishing crosslinkages brought by saccharide aldehydes. The results show that the keratin fibers after crosslinking with saccharide aldehydes had substantially higher stress retention after soaked in water for a week than that of fibers without aldehyde crosslinkages. High retention of fiber strength after crosslinking demonstrated the feasibility to apply feathers in textiles and other fiber-based industries on a large scale. At room temperature, stress retention crosslinked fibers by saccharide aldehyde can be as high as $92 \%$ and the stress retention of such fibers at $35{ }^{\circ} \mathrm{C}$ was still above $85 \%$. The reason is that the substantially strengthened interaction between crosslinked protein

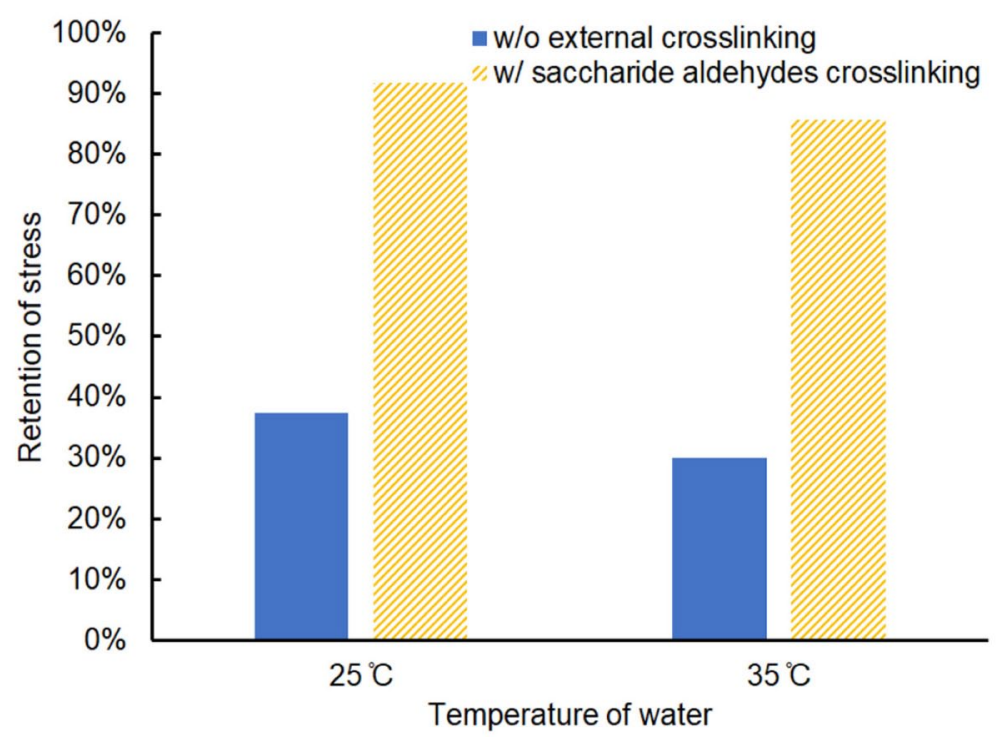

Fig. 6. Effect of crosslinking using disaccharide-tetra-aldehydes on property retention of keratin fibers immersed in water for 1 week (The crosslinking was conducted under $50^{\circ} \mathrm{C}$ for $1 \mathrm{~h}$ with aldehyde concentration of $0.52 \mathrm{M}$ ). 
molecules increased the stability of fibers in water and largely kept the morphology of fibers. Also, the hydrophilicity of the protein molecules after crosslinking decreases. Therefore, stress retention of fibers remarkably improved.

\subsection{Comparison in wet properties with known common fibers}

Table 1 compares the properties of keratin fibers with various treatments to wool. Results show that crosslinking using saccharide aldehydes can substantially increase the mechanical properties of keratin fibers and suitable for fiber production on a continuous spinning line. Breaking stress, strain and toughness of fibers at dry state after crosslinking using saccharide aldehydes increased around 55\%,70\% and $50 \%$, respectively. Breaking stress, strain and toughness at wet state of fibers after crosslinking using saccharides aldehydes increased $100 \%$, $30 \%$ and $25 \%$. Above properties of fibers were comparable to wool. Crosslinking using saccharide aldehydes was better at improving the mechanical properties of keratin fibers than that using citric acid from Table 1. Possible reasons are different conditions for crosslinking using citric acid and saccharide aldehydes. Crosslinking using citric acid needed weak alkalinity, while crosslinking using saccharide aldehyde took place under acidic conditions [30]. On continuous spinning lines, fibers were more stable in acidic conditions. Secondary structures of fiber under alkali spinning conditions might be damaged.

Table 1 Comparison of mechanical properties of crosslinked keratin fibers to wool fibers.

\begin{tabular}{|c|c|c|c|c|c|c|}
\hline \multirow[t]{2}{*}{ Fiber source } & \multicolumn{3}{|l|}{ Dry state } & \multicolumn{3}{|l|}{ Wet state } \\
\hline & $\begin{array}{l}\text { Strength } \\
(\mathrm{MPa})\end{array}$ & Strain & $\begin{array}{l}\text { Toughness } \\
\left(\mathrm{J} \mathrm{cm}^{-3}\right)\end{array}$ & $\begin{array}{l}\text { Strength } \\
(\mathrm{MPa})\end{array}$ & Strain & $\begin{array}{l}\text { Toughness } \\
\left(\mathrm{J} \mathrm{Cm}^{-3}\right)\end{array}$ \\
\hline Keratin fibers [17] & $110-145$ & $8-12 \%$ & $17-19$ & $60-90$ & $22-28 \%$ & $22-28$ \\
\hline $\begin{array}{l}\text { Keratin fibers crosslinked } \\
\text { with sucrose derivatives }\end{array}$ & $195-215$ & $16-22 \%$ & $27-32$ & $120-140$ & $27-35 \%$ & $29-35$ \\
\hline Wool [31] & 150-190 & $30-40 \%$ & $28-38$ & $120-160$ & $42-50 \%$ & $33-40$ \\
\hline
\end{tabular}




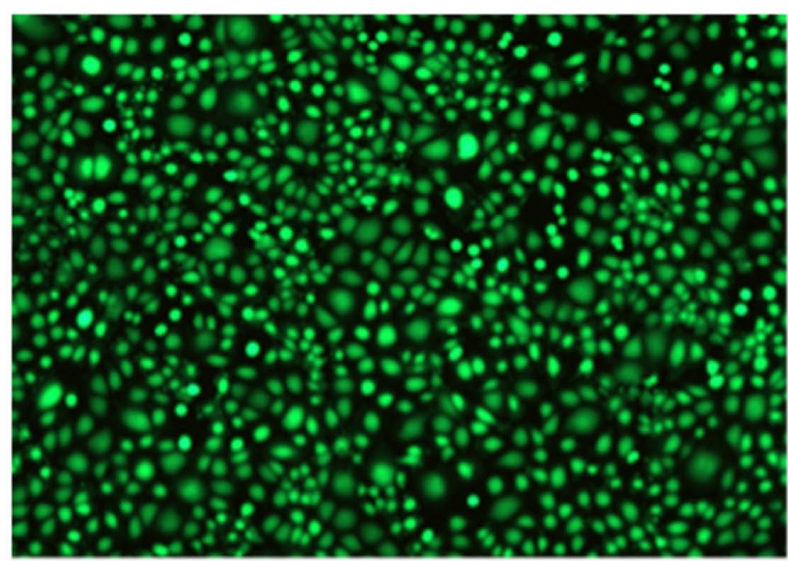

Blank

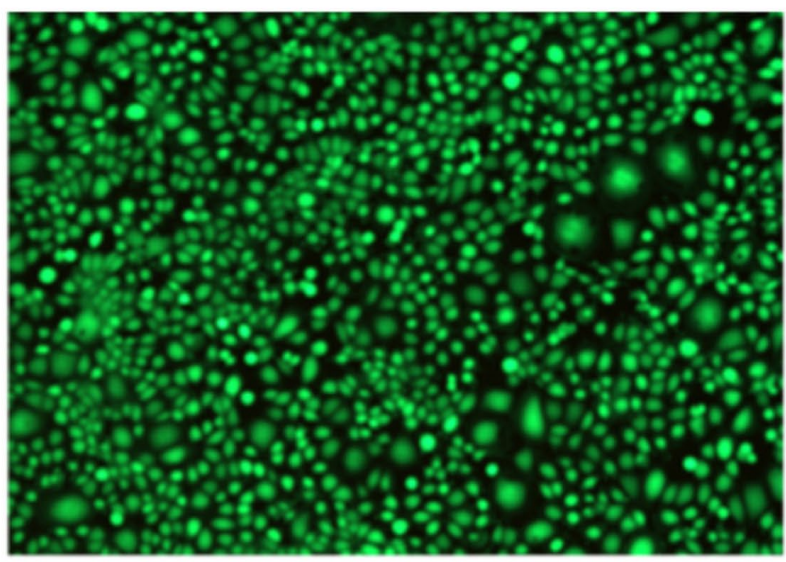

w/o aldehyde crosslinking

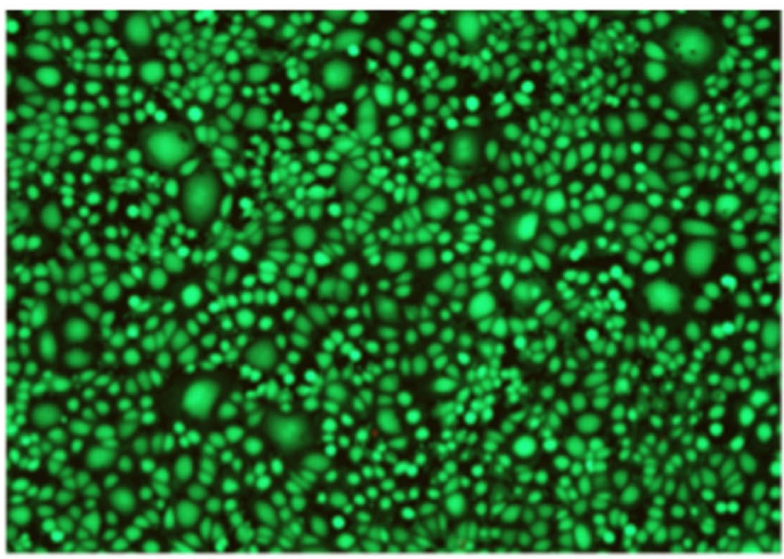

cr with disaccharide-tetra-aldehydes

\subsection{Cytocompatibility}

Fig. 7 demonstrates keratin crosslinked with formyl-saccharides had good biocompatibility with NIH3T3 cells. As shown in Fig. 7, the NIH3T cells on keratin fibers with saccharidealdehydes crosslinking were not lower than keratin without crosslinking and blank. The high viability and large numbers of cells in keratin with saccharidealdehyde crosslinking indicated that crosslinkages formed by saccharide-aldehyde did not decrease the biocompatibility of keratin. The reason for this is the complete elimination of toxic formaldehyde in preparation of crosslinkers.

Fig. 7. (a) Viability of NIH3T3 cells after 3 days of culture on keratin without sucrosetetra-aldehydes crosslinking. Live and dead cells are green and red, respectively. 
Table 2 Color of keratin fibers with crosslinkages from saccharide-aldehydes.

\begin{tabular}{lccc} 
Keratin fibers & $L^{*}$ & Yellowness Index & Delta $E_{c m c}$ \\
\hline w/o saccharide-aldehydes & 86.7 & 20.1 & \\
w/ saccharide-aldehydes & 87.1 & 20.3 & 0.56
\end{tabular}

The lightness value, $L^{*}$, represents the darkest black at $L^{*}=0$, and the brightest white at $L^{*}=$ 100. Yellowness was based on ASTM E 313

\subsection{Good color retention}

Color retention of protein fibers after crosslinking is another critical factor to be evaluated. Table $\mathbf{2}$ demonstrates excellent color retention of saccharide-aldehydes crosslinked keratin fibers. The color of the aldehyde-crosslinked fibers, L value, and yellowness were almost identical without aldehyde-crosslinking. The color difference between the above two fibers, illustrated as $\mathrm{DE}_{\mathrm{CMC}}$ was only 0.56 , indicating no significant difference between the above two fibers. Usually, the values less than 1 , determined by $D E_{C M C^{\prime}}$ indicates no color difference.

\subsection{Low consumption of amine groups}

Table 3 compares the consumption of amine groups on keratin among various green crosslinking approaches. Based on the amounts used in previous work for good wet stability of protein products, previous crosslinking consumed the majority or completed amine groups of protein, making the dyeing of regenerated fibers impossible. Because of the low reactivity of citric acid, an excessive amount of citric acid was added into protein materials. Though many

Table 3 Consumption of amine groups from various crosslinking methods.

Crosslinking methods

Citric acid at high temperature [32]

Citric acid at low temperature [25]

Sugar-aldehydes high-temperature [23]

Sugar-aldehydes without quality control [22]

This work
Consumption of amine groups on protein *
$100 \%$
$100 \%$
$100 \%$
$79 \%$
$31 \%$

* Calculation of consumption was based on that one functional group on crosslinkers consumed one amine group on protein. 
citric acid molecules would not form crosslinkages in protein, those citric acid molecules still consume amine groups of protein molecules. Nevertheless, this work only consumed $30 \%$ of amine groups theoretically, providing sufficient dyeing sites in regenerated keratin fibers.

\subsection{Environmental and economic benefits}

Fig. S1 shows data of fiber production globally and potential production volumes from waste keratinous materials including chicken feathers. Results show that transformation from keratinous materials to high-quality fibers can substantially alleviate the dependence of textile fibers on petroleum-based products and promote the sustainable development of fiber industry. As a result, the impact of fiber industry on the environment can be remarkably reduced. Given that more than 10 million tons of keratinous materials are generated annually, keratin fiber addition from such wastes can reduce the market share of petroleum-based fibers from $70 \%$ to $60 \%$ without using additional land, water, and other natural resources for cultivation. As a result, the addition of fibers from keratinous wastes can reduce the burden of discharge and recycling petroleum-based fibers by around 10 million tons per year. Such addition can have a positive impact on the environment. In addition, to establish a chicken feather based biofiber industry will also greatly contribute to the safety and sustainability of the agricultural and food industries.

Table S1 demonstrates that restoration of chicken feathers to fibers similar to wool in terms of stretchability and wet stability could better promote sustainable transference and decontamination of hazardous poultry by-products. Given that the similar properties between regenerated fibers and wool, keratin fibers could be sold at the same price as wool. The potential value addition from keratin fibers from chicken feathers can be up to 35 billion dollars per year, 30 and 17 times higher than that from biofertilizers and animal feed, respectively. The potential production of protein fibers from chicken feathers is already 2.5 times higher than the current output of both wool and silk. 


\section{Conclusions}

Formaldehyde-free sucrose-tetra-aldehydes have been successfully applied to a continuous spinning line for the clean production of keratin fibers with excellent water-resistance and mechanical properties. With fine control of length of crosslinkages, use of crosslinkers was minimized, toughness and wet strength of fibers from continuous spinning line increased $50 \%$ and $100 \%$, respectively. The retention of fiber tenacity was as high as $92 \%$ after immersed in water for 1 week. The color of fibers did not change, and fibers were dyeable after crosslinking. Transformation of feather wastes to protein fibers can help the sustainable decontamination of poultry wastes and reduce the demand for petroleum-based fibers by 100 million tons per year globally. As a result, environmental pressure will be substantially alleviated. In addition, the successful production of protein fibers can add values of around 35 billion dollars to the poultry industry.

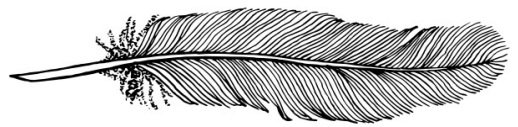

Supplementary materials One supplemental figure (Fig. S1) and one supplemental table (Table S1) follow the References. A demonstration video is attached to the archive record for this article.

Acknowledgments The research has been supported by the United States Department of Agriculture National Institute of Food and Agriculture (award number 201967021-29940), the Nebraska Environmental Trust (NET Grant 18-116) and the Agricultural Research Division at the University of Nebraska-Lincoln. The research was performed in part in the Nebraska Center for Materials and Nanoscience, which are supported by the National Science Foundation under Award ECCS: 2025298, and the Nebraska Research Initiative. Bingnan is grateful to the John and Louise Skala Fellowship and AATCC Students Grant for their financial support. Faqrul is grateful to Dr. Joan Laughlin Fellowship.

Competing interests There are no conflicts to declare. 


\section{References}

[1] T. Tesfaye, B. Sithole, D. Ramjugernath, Valorisation of waste chicken feathers: optimisation of decontamination and pre-treatment with bleaching agents using response surface methodology, Sustain. Chem. Pharm. 8 (2018) 21-37, https://doi.org/10.1016/j.scp.2018.02.003

[2] V.K. Gaur, P. Sharma, R. Sirohi, M.K. Awasthi, C.-G. Dussap, A. Pandey, Assessing the impact of industrial waste on environment and mitigation strategies: a comprehensive review, J. Hazard. Mater. 398 (2020), 123019, https://doi. org/10.1016/j.jhazmat.2020.123019

[3] V. McGovern, Recycling poultry feathers: more bang for the cluck, Environ. Health Perspect. 108 (2000) A366-A369.

[4] M. Ewall, Air Pollution and Toxic Hazards Associated with Poultry Litter Incineration, Justice Network: Philadelphia, PA, USA, 2007.

[5] J.D. Miller, The role of dust mites in allergy, Clin. Rev. Allergy Immunol. 57 (2019) 312-329, https://doi.org/10.1007/s12016-018-8693-0

[6] S. Health, Executive, Statement of Evidence: Respiratory Hazards of Poultry Dust, Health and Safety Executive 03/09, Suffolk, UK, 2008.

[7] Q. Li, Progress in microbial degradation of feather waste, Front. Microbiol. 10 (2019) 2717, https://doi.org/10.3389/fmicb.2019.02717

[8] Y. Han, M. Ma, N. Li, R. Hou, C. Huang, Y. Oda, Z. Wang, Chlorination, chloramination and ozonation of carbamazepine enhance cytotoxicity and genotoxicity: multi-endpoint evaluation and identification of its genotoxic transformation products, J. Hazard. Mater. 342 (2018) 679-688, https://doi. org/10.1016/j.jhazmat.2017.08.076

[9] G. Gupta, W. Gardner, Use of clay mineral (montmorillonite) for reducing poultry litter leachate toxicity $\left(\mathrm{EC}_{50}\right)$, J. Hazard. Mater. 118 (2005) 81-83, https://doi.org/10.1016/j.jhazmat.2004.05.024

[10] B. Mu, W. Li, H. Xu, L. Xu, Y. Yang, Freeze-extrusion for controllable assembly of 3- dimensional ultra-fine and amorphous fibrous matrices: potential applications in sorption, J. Mater. Chem. A 6 (2018) 10320-10330, https://doi. org/10.1039/C8TA01845F

[11] Y. Ren, J. Gong, R. Fu, J. Zhang, K. Fang, X. Liu, Antibacterial dyeing of silk with prodigiosins suspention produced by liquid fermentation, J. Clean. Prod. 201 (2018) 648-656, https://doi.org/10.1016/j.jclepro.2018.08.098

[12] B. Mu, F. Hassan, Q. Wu, Y. Yang, Ductile keratin/deacetylated chitin composites with nanoparticle-induced formation of ordered and entangled structures, Compos. Sci. Technol. 200 (2020), 108462, https://doi. org/10.1016/j.compscitech.2020.108462

[13] X. Liu, Y.-L. Hsieh, Tunable surface wettability and pH-responsive 2D structures from amphiphilic and amphoteric protein microfibrils, RSC Adv. 10 (2020) 33033-33039, https://doi.org/10.1039/d0ra05067a 
[14] D. Nataraj, S. Sakkara, M. Hn, N. Reddy, Properties and applications of citric acid crosslinked banana fibre-wheat gluten films, Ind. Crop. Prod. 124 (2018) 265-272, https://doi.org/10.1016/j.indcrop.2018.07.076

[15] X. Fei, W. Wei, F. Zhao, Y. Zhu, J. Luo, M. Chen, X. Liu, Efficient toughening of epoxy-anhydride thermosets with a biobased tannic acid derivative, ACS Sustain. Chem. Eng. 5 (2017) 596-603, https://doi.org/10.1021/ acssuschemeng.6b01967

[16] Y. Wang, L. Chen, H. Cheng, B. Wang, X. Feng, Z. Mao, X. Sui, Mechanically flexible, waterproof, breathable cellulose/polypyrrole/polyurethane composite aerogels as wearable heaters for personal thermal management, Chem. Eng. J. 402 (2020), 126222, https://doi.org/10.1016/j.cej.2020.126222

[17] B. Mu, F. Hassan, Y. Yang, Controlled assembly of secondary keratin structures for continuous and scalable production of tough fibers from chicken feathers, Green Chem. 22 (2020) 1726-1734, https://doi.org/10.1039/ C9GC03896E

[18] B. Mu, H. Xu, W. Li, L. Xu, Y. Yang, Spinnability and rheological properties of globular soy protein solution, Food Hydrocoll. 90 (2019) 443-451, https:// doi.org/10.1016/j.foodhyd.2018.12.049

[19] K. Gregg, G.E. Rogers, Feather Keratin: Composition, Structure and Biogenesis, Biology of the Integument, Springer, 1986, pp. 666-694, https:// doi.org/10.1007/978-3-662-00989-5 33

[20] Bingnan Mu, Qianmei Wu, Lan Xu, Yiqi Yang, A sustainable approach to synchronous improvement of wet-stability and toughness of chitosan films, Food Hydrocoll. 123 (2022), 107138, https://doi.org/10.1016/j. foodhyd.2021.107138

[21] Bingnan Mu, Lan Xu, Yiqi Yang, Flexible and wet stable starch films crosslinked with sugar-based aldehydes, Ind. Crops. Prod. 173 (2021), https:// doi.org/10.1016/j.indcrop.2021.114109

[22] X. Mi, Y. Chang, H. Xu, Y. Yang, Valorization of keratin from food wastes via crosslinking using non-toxic oligosaccharide derivatives, Food Chem. 300 (2019), 125181, https://doi.org/10.1016/j.foodchem.2019.125181

[23] L. Liu, Z. Chen, B. Wang, Y. Yang, Improving wet strength of soy protein films using oxidized sucrose, J. Appl. Polym. Sci. 132 (2015), https://doi. org/10.1002/app.41473

[24] R. Reddy, Q. Jiang, P. Aramwit, N. Reddy, Litter to leaf: the unexplored potential of silk byproducts, Trends Biotechnol. (2020), https://doi. org/10.1016/j.tibtech.2020.11.001

[25] N. Reddy, Y. Li, Y. Yang, Alkali-catalyzed low temperature wet crosslinking of plant proteins using carboxylic acids, Biotechnol. Prog. 25 (2009) 139-146, https://doi.org/10.1002/btpr.86

[26] J. Bobbitt, Periodate oxidation of carbohydrates, Adv. Carbohydr. Chem. 11 (1956) 1-41, https://doi.org/10.1016/S0096-5332(08)60115-0 
[27] W. Chen, G.C. Lickfield, C.Q. Yang, Molecular modeling of cellulose in amorphous state part II: effects of rigid and flexible crosslinks on cellulose, Polymer 45 (2004) 7357-7365, https://doi.org/10.1016/j.polymer.2004.08.023

[28] N. Reddy, K. Warner, Y. Yang, Low-temperature wet-cross-linking of silk with citric acid, Ind. Eng. Chem. Res. 50 (2011) 4458-4463, https://doi. org/10.1021/ie102226f

[29] Y.-Q. Yang, S. Li, Silk fabric non-formaldehyde crease-resistant finishing using citric acid, J. Text. Inst. 84 (1993) 638-644, https://doi. org/10.1080/00405009308658995

[30] H. Xu, L. Shen, L. Xu, Y. Yang, Low-temperature crosslinking of proteins using nontoxic citric acid in neutral aqueous medium: mechanism and kinetic study, Ind. Crop. Prod. 74 (2015) 234-240, https://doi.org/10.1016/j. indcrop.2015.05.010

[31] N. Reddy, Y. Yang, Innovative Biofibers from Renewable Resources, Springer, Berlin, Heidelberg, 2015.

[32] Y. Yang, N. Reddy, Properties and potential medical applications of regenerated casein fibers crosslinked with citric acid, Int. J. Biol. Macromol. 51 (2012) 37-44, https://doi.org/10.1016/j.jijbiomac.2012.04.027 
Worldwide production volumein 1,000 metric tons

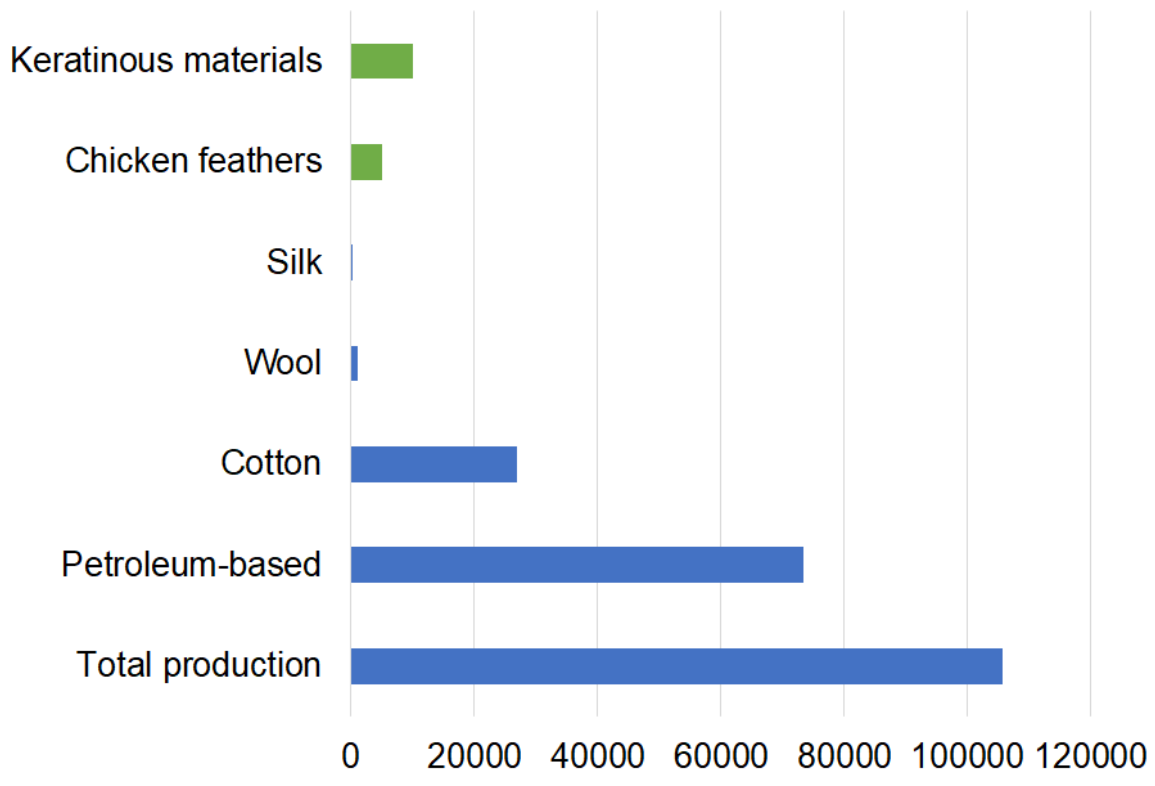

Figure 1 Comparison of annual worldwide production volume of fibers and keratinous wastes. The data from followings work ${ }^{1,2}$. 
Table 1. Potential value additions to poultry industry from various end keratinous products

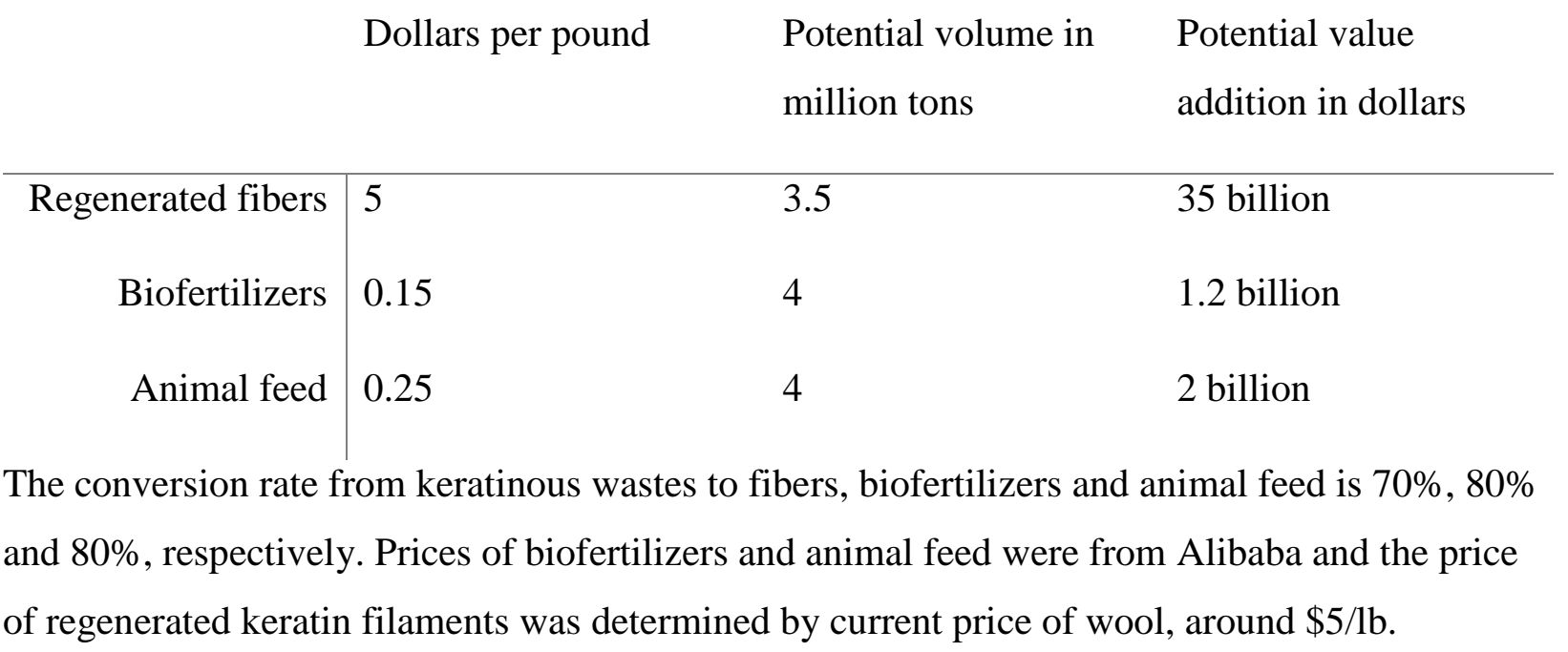

1. Statista Worldwide production volume of chemical and textile fibers from 1975 to 2018

https://www.statista.com/statistics/263154/worldwide-production-volume-of-textile-fibers-since-1975/ (accessed 4/17).

2. Shahbandeh, M. Global cotton production volume from 1990 to 2018.

https://www.statista.com/statistics/259392/cotton-production-worldwide-since-1990/ (accessed 4/17). 